Egregi direttori,

Rileggendo il mio articolo, pubblicato nel Vol. IV, 1 (1983) di Quaderni d'italianistica, ho notato un mio errore di battitura a macchina riprodotto sul periodico. Parlo della nota numero 19 (p. 110 ), in cui appaiono le parole "pascoliano" e "Pascoli alla rovescia." Le parole usate dal Professor A. Vicari, dal cui saggio "I miti di Luigi Pirandello" citavo, sono "pascaliano" e "Pascal alla rovescia." Chiedo scusa al Professor Vicari per la svista.

\title{
C. Federici
}

\section{Comunicato AISLLI}

L'Associazione Internazionale per gli Studi di Lingua e Letteratura Italiana (AISLLI), terrà il XII congresso in Canada dal 6 al 10 maggio 1985 sul tema "Letteratura italiana e arti figurative"; una seduta particolare sarà dedicata all'insegnamento dell'italiano in Canada. Sedi dei lavori saranno le città di Toronto, Hamilton e Montreal.

Il programma prevede dodici relazioni presentate nel corso delle sedute plenarie ed una sessantina di comunicazioni (di circa 15/20 minuti ciascuna) presentate nei gruppi specializzati.

In particolare verranno discussi i seguenti temi: generi letterari e generi figurativi (paesaggio, natura morta, ritratto, ecc.); I'occhio come metafora costruttiva del testo; il libro illustrato (l'illustrazione come interpretazione del testo); poesia visiva, la parola figurata, "calligrammes"; il "non finito" in arte e in letteratura; le avanguardie assimilatrici (la convergenza d'arte e di letteratura nelle poetiche delle avanguardie); narrazione e descrizione, reciprocità di modelli figurativi e letterari; il cinema e la letteratura.

La lingua ufficiale sarà l'italiano.

Quanti sono interessati a presentare una comunicazione devono far pervenire entro il 31 dicembre 1984 un riassunto di una cartella in due copie al Prof. Antonio D'Andrea, Department of Italian, McGill University, 1001 Sherbrooke West, Montréal, Quebec, Canada, H3A 1 G5.

Per informazioni sul congresso stesso ci si può rivolgere al Prof. Antonio Franceschetti, Division of Humanities, Scarborough College, University of Toronto, West Hill, Ontario, Canada, M1C 1A4 tel. (416) 284-3307. 